\title{
Miniature and MOEMS Flow Sensors
}

\author{
by
}

\author{
D. Fourguette, D. Modarress, F. Taugwalder \\ D. Wilson ${ }^{1}$, M. Koochesfahani ${ }^{2 *}$, M. G harib ${ }^{2}$ \\ VioSense Corporation \\ 36 S. Chester Ave., Pasadena, CA 91106
}

\begin{abstract}
Recent progress in the development of a miniature Laser D oppler Anemometer (LDA) and a micro optical shear stress sensor is described. Miniaturization of these sensors has been achieved with the use of integrated optics and micro fabrication techniques. This paper describes the fabrication of the two sensors and presents an experiment for the evaluation of the sensors. The results show perfect agreement between the boundary layer velocity gradient performed with the LDA, and the measurements obtained with the shear stress sensor. The range of experimental conditions suitable for the wall shear sensor is reported. Finally, we describe the application of the sensors in a series of tests performed at the William B. Morgan Large Cavitation Channel of the Navy's Carderock Division, in Memphis, Tennessee.
\end{abstract}

\section{Introduction}

Laser Doppler anemometry (LDA) has been in use as a research tool for over three decades. Since its inception in 1964 by Yeh and Cummins ${ }^{1}$, the LDA technique has matured to the point of being well understood and, when used carefully, providing accurate experimental data. LDA's unique attributes of linear response, high frequency response (defined by the quality of the seed particles) and non-intrusiveness have made it the technique of choice for the study of complex multi-dimensional and turbulent flows 2,3 . It has also been modified for use in multi-phase flows ${ }^{4}$, particle sizing 5 , and remote sensing ${ }^{6}$, to cite a few examples. Advancements in the areas of laser performance, fiber optics and signal processing have enhanced the utility of the technique in fluid mechanics and related research areas.

The overall configuration of LD A systems has not been altered over the period of its existence and as such, its use has been limited to laboratories, experimentally unique events, and a modest number of industrial applications. To expand the utility of the LD A to other applications with the need for speed measurement, D oppler-based sensors that are smaller, integrated, and easier to use need to be developed.. These compact sensors can then be embedded into models, and maintain a fixed working distance throughout the tests. The results of our on-going efforts in this direction are described here.

Recent advances in micro machining ${ }^{7}$ have prompted the development of micro sensors; such as pressure, proximity, and wall shear stress to name a few. These sensors are small, non-intrusive, and can be embedded in a model for spatially resolved real time in-situ measurements. Micro machined wall shear stress sensors recently fabricated are either

${ }^{1}$ NASA Jet Propulsion Laboratory, Pasadena, CA

2 California Institute of Technology, Pasadena, CA

* On leave from Michigan State University, East Lansing, MI

Copyright@2001 by the American Institute of A eronautics and Astronautics, Inc. All rights reserved 
mechanical using a floating element, thermal using heat dissipation, or optical using an interference fringe pattern at the surface of the model ${ }^{8}$. The optical wall shear stress sensor described here uses a technique developed by Naqwi and Reynolds ${ }^{9}$ who measured the velocity gradient within the first hundred microns above the wall using a diverging fringe pattern originating at the wall. In that arrangement, the velocity and the fringe spacing increase proportionally with the distance from the wall. This technique is akin to LDA in its concept and processing. This method yields accurate results as long as the measurements are conducted within the linear sub-layer. The development and fabrication of wall shear stress sensor described here utilize recent advances in optical MEMS technology (MOEMS) unavailable fifteen years ago. As a result, the large optical setup used by Naqwi and Reynolds to generate optical fringes is now reduced to a sensor 15 $\mathrm{mm}$ in diameter and $15 \mathrm{~mm}$ long, which is easily embeddable into a model ${ }^{10}$. The conceptual design and fabrication are described in this paper.

The combined results from the miniature LD A and the shear stress sensor are presented here. The measurements were conducted in a laminar boundary layer and a perfect agreement was found between the velocity gradient close to the wall measured with the LDA and the velocity gradient measured with the shear stress sensor. Experimental conditions for laminar and turbulent boundary layers at which the shear stress sensor will provide accurate results are also reported.

\section{The miniature LDA}

The miniature $\mathrm{LDA}^{1}$ sensor described here was the precursor to the micro-systems under development. The mini-LDA used in the present experiment is shown in Figure 1. The LD A unit contains the light source, miniature optics, receiving optics, and detection system. In our present design, the diode laser does not

${ }^{1}$ Patent pending require temperature stabilization. The miniature LDA is mounted onto a motorized traverse as to position the probe volume at precise locations (with one micron resolution) over $33 \mathrm{~mm}$ travel above the flow model surface. The assembly is enclosed in a watertight cavity with a dimension of $40 \mathrm{~mm}$ x $60 \mathrm{~mm}$ x $115 \mathrm{~mm}$. The frequency shifting range was $2 \mathrm{MHZ}$. The miniature LD A sensor specifications are given in Table 1.

\begin{tabular}{|l|l|}
\hline Working distance & 0 to $33 \mathrm{~mm}$ \\
\hline Laser wavelength & $785 \mathrm{~nm}$ \\
\hline Probe volume dimensions & $\begin{array}{l}15 \times 20 \times 90 \\
\mu \mathrm{m}^{3}\end{array}$ \\
\hline Fringe separation & $1.27 \mu \mathrm{m}$ \\
\hline $\begin{array}{l}\text { Number of fringes in probe } \\
\text { volume }\end{array}$ & 14 \\
\hline Frequency shifting & $2 \mathrm{MHz}$ \\
\hline
\end{tabular}

Table 1. LDA specifications.

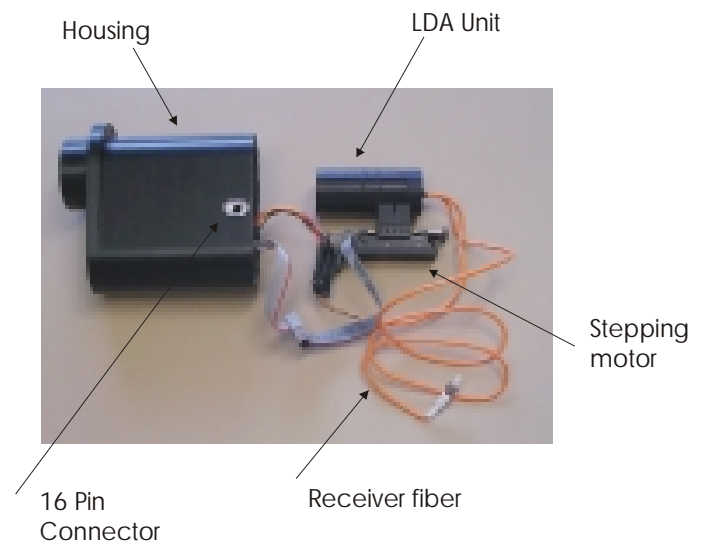

Figure 1. Photograph of the miniature LDA, traversing mechanism and the enclosure.

\section{The wall shear stress sensor Principle of operation}

The optical shear stress sensor developed for this experiment is based on a technique first presented by Naqwi and Reynolds using conventional optics. They projected a set of diverging fringe patterns and used the Doppler shift to measure the gradient of the flow velocity at the wall.

Figure 2 shows a schematic of the MOEMS 
wall shear stress sensor principle. Diverging interference fringes originate at the surface and extend into the flow. The scattered light from the particle passing through the fringes is collected through a window at the surface of the sensor. The region defined by the intersection of the transmitter and receiver fields was centered at approximately $66 \mu \mathrm{m}$ above the surface and measured about $30 \mu \mathrm{m}$ high.

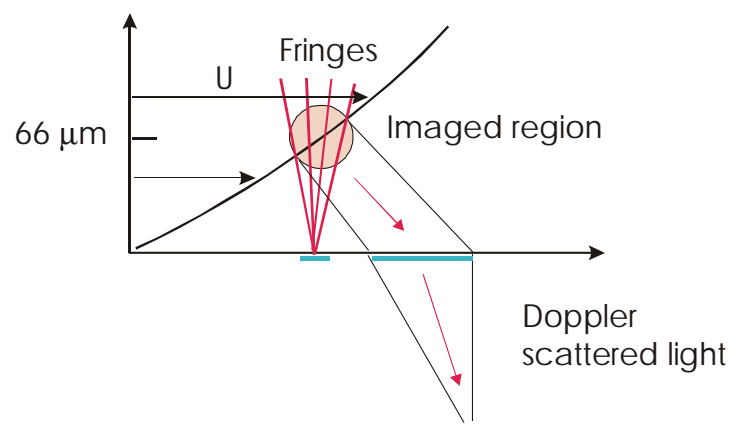

Figure 2. Schematic of the optical shear stress sensor principle of measurement.

The local fringe separation, $\delta$, was designed to be linear with the distance from the sensor, $\mathrm{y}$, given by $\delta=k \times y$, where k is the fringe divergence rate. As particles in the fluid flow through the linearly diverging fringes, they scatter light with a frequency $f$ that is proportional to the instantaneous velocity and inversely proportional to the fringe separation at the location of particle trajectory as shown in Figure 2. The velocity of the particle is therefore $u=f \times \delta$. The D oppler frequency simply multiplied by the fringe divergence yields the velocity gradient

$$
\frac{u}{y}=f \times k
$$

which is equal to the wall shear,

$$
\sigma=\left.\frac{\partial u}{\partial y}\right|_{w} \equiv \frac{u}{y}
$$

in the quasi linear sub-layer region of the boundary layer. The signal conditioning and processing required for the shear stress sensor is identical to those used for the LDA instrument.

The primary difference between the Laser Doppler Anemometer and the micro- shear stress sensor is that the former uses a set of parallel fringes at the probe volume for the measurement of velocity, while the shear stress sensor uses a set of diverging fringes to measure the gradient of the velocity.

\section{Fabrication}

A conceptual drawing of the micro-shear stress sensor is shown in Figure 3. The light output of a mono-mode optical fiber was allowed to diverge onto a PMMA diffractive lens ${ }^{11,12}$ and spatially filtered through two parallel slits. The output was a diverging fringe system slightly slanted to the vertical axis. The slant placed the probe volume above a window to allow for more efficient light gathering by the receiver PMMA diffractive lens. The light was imaged onto a fiber coupled to a photodiode.

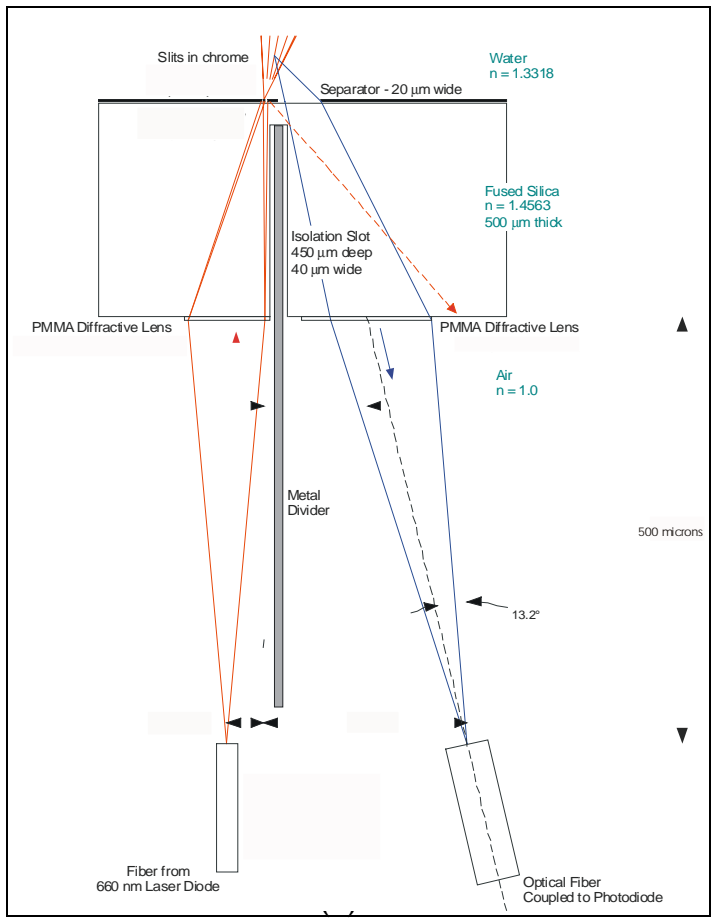

Figure 3. Schematic of the shear stress sensor

The fringe separation was measured with an 
imaging instrument. Figure 4 shows a photograph of fringes 150 microns above the sensor. The fringe separation at different distances was measured. The divergence rate of the fringes was measured to be linear with a slope in close agreement with the design value.

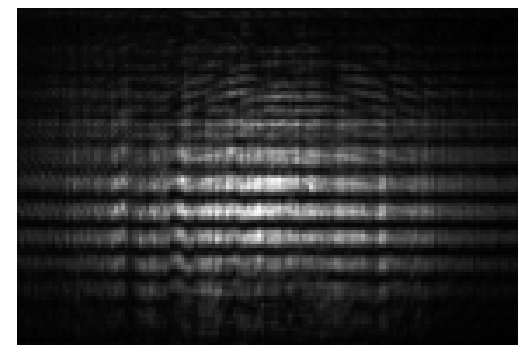

Figure 4. Fringes from the slits output.

A photograph of the shear stress sensor is shown in Figure 5. The sensor substrate, shown in Figure 3, is mounted into the sensor element location shown on the front face of the assembly. The diverging fringe pattern is illuminated with the help of a fogger.

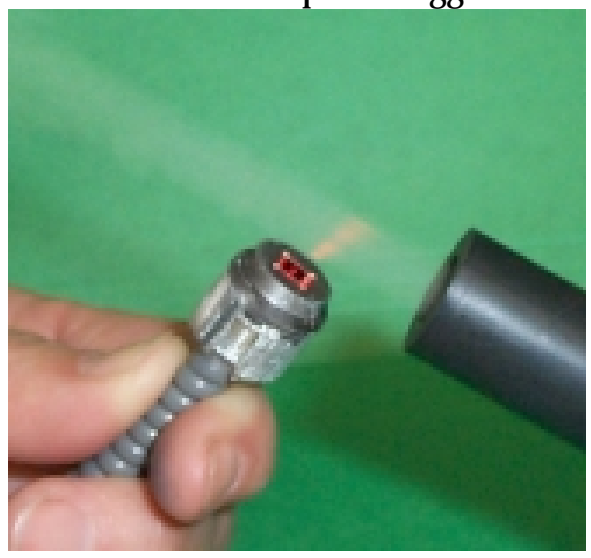

Figure 5. Shear stress sensor mount.

\section{Results}

\section{Description of the experiment}

The miniature LDA and the shear stress sensor were tested in a water tunnel facility at the $\mathrm{G}$ raduate Aeronautics Laboratory of the California Institute of Technology. The facility is equipped with a $90 \mathrm{~cm}$ long test section in which different model geometries can be inserted. The miniature LDA and the shear stress sensors were mounted on a flat plate at $\mathrm{x}=34.93 \mathrm{~cm}$ and $39.37 \mathrm{~cm}$ from the leading edge, as shown in Figure 6.

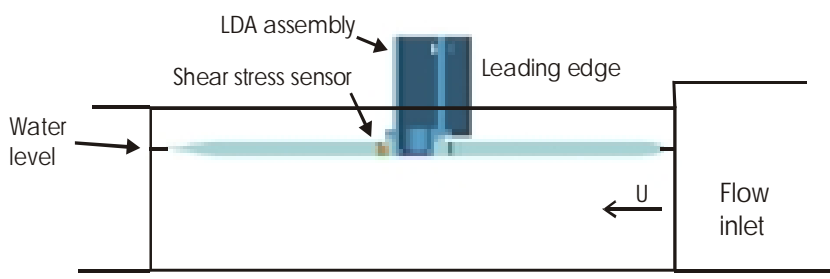

Figure 6. Schematic of the test section with the flat plate.

Measurements were made at free-stream velocities of $\mathrm{U}_{0}=18.1$ and $\mathrm{U}_{0}=26.9 \mathrm{~cm} / \mathrm{s}$. Velocity surveys were conducted and the data are shown in Figure 7 and Figure 8. Frequency shifting was not used for these measurements. A Blasius profile was fitted to the data. The fit agreed well with the velocity survey. Closer examination of the data indicated that the measured velocity at the point closest to the wall was slightly larger than the corresponding value for the Blasius profile. The same conclusion was arrived at for both run cases. This variation was of important consequence when the results of the micro shear stress was compared with the wall shear estimated from the velocity profile.

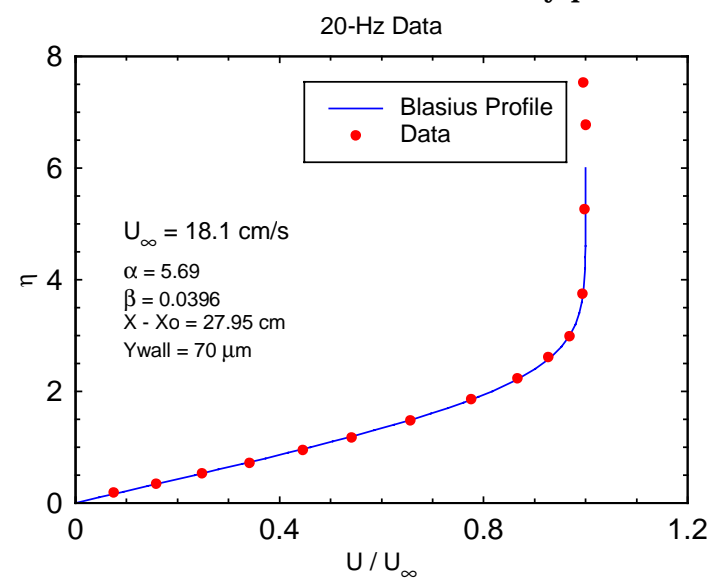

Figure 7. Results of the velocity survey using the miniature $L D A$ for $U_{0}=18.1 \mathrm{~cm} / \mathrm{s}$. 


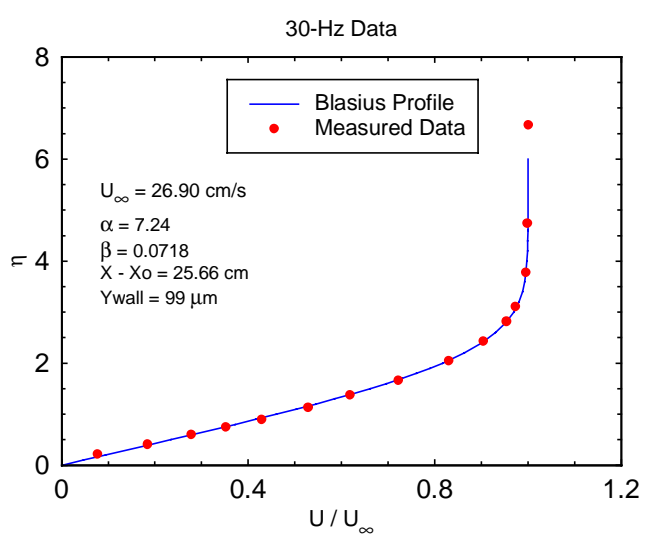

Figure 8. Results of the velocity survey using the miniature LDA for $U_{0}=26.9 \mathrm{~cm} / \mathrm{s}$.

Shear stress measurements were conducted at the same experimental conditions as that for the velocity surveys. Figure 9 and Figure 10 show histograms of the instantaneous D oppler frequencies obtained for the $\mathrm{U}_{0}=18.1$ $\mathrm{cm} / \mathrm{s}$ and $\mathrm{U}_{0}=26.9 \mathrm{~cm} / \mathrm{s}$ cases respectively. The velocity gradients were estimated from the ensemble average of the instantaneous values.

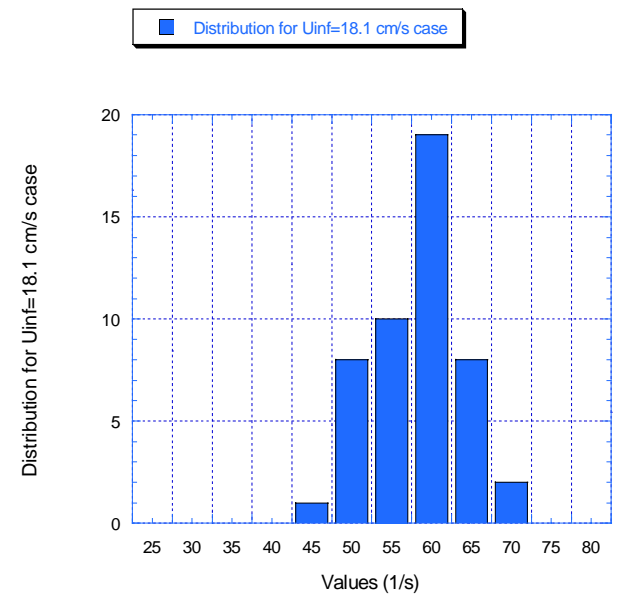

Figure 9. Histogram of the velocity gradient measurements for the $\mathrm{U}_{0}=18.1 \mathrm{~cm} / \mathrm{s}$ case.

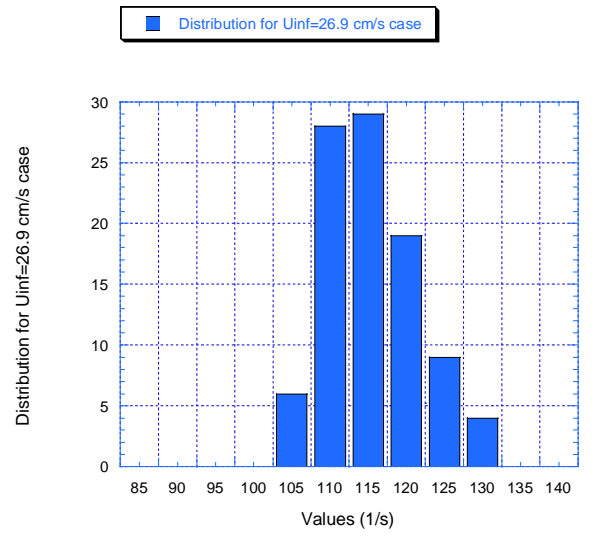

Figure 10. Histogram of the velocity gradient measurements for the $U_{0}=26.9 \mathrm{~cm} / \mathrm{s}$ case.

The data obtained from the micro- shear stress was compared with the wall shear estimated from the velocity profiles. The wall velocity gradient was estimated using two methods. The first method involved calculating the velocity gradient at the wall using the last two data points of the velocity survey and the second method involved obtaining the velocity gradient at the wall using the curve fit. Figure 11 shows the comparison of wall velocity gradients. The gradient obtained from the slope of the velocity data agrees well with the micro-shear stress data. However both gradients were greater than that calculated from the Blasius fit. This result led us to postulate the presence of a slightly favorable pressure gradient at the measurement location.

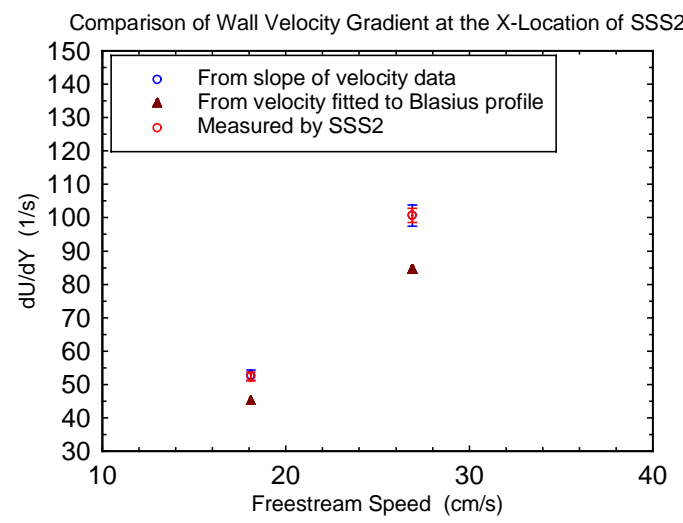

Figure 11. Comparison of wall velocity gradients obtained with the miniature LDA and the micro-shear stress sensor. 
Ideally, accurate measurement of the wall shear stress with the micro-shear stress sensor requires that the probe volume to be within the linear region of the boundary layer. An estimate of the measurement errors as function of the flow Reynolds number was calculated for the flow on a flat plate in the absence of a pressure gradient for this particular shear stress sensor. Figure 12 shows the error bounds for a laminar boundary layer and Figure 13 shows the results for a turbulent boundary layer.

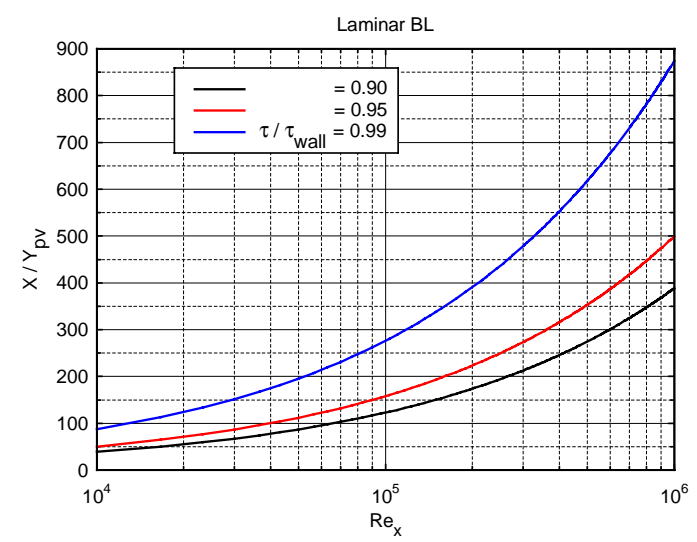

Figure 12. Normalized height above the plate versus Reynolds number.

In both plots, $Y_{p v}$ represents the location of the probe volume above the surface. The accuracy of the measurement is better than 99\% for experimental conditions above the blue line. For instance, the sensor described in this paper ( $\mathrm{Y}_{\mathrm{pv}}=66$ microns) will yield accurate results for a Reynolds number $=10^{6}$ when located $1 \mathrm{~m}$ downstream from the leading edge of the plate. The error plots show that for turbulent flows, the measurement errors increase rapidly with the Reynolds number. Therefore, it is important that the sensor to be fabricated such that the probe volume remain within the laminar sublayer at all times. New shear stress sensor designs are currently under development to extend the operating envelope of these sensors.

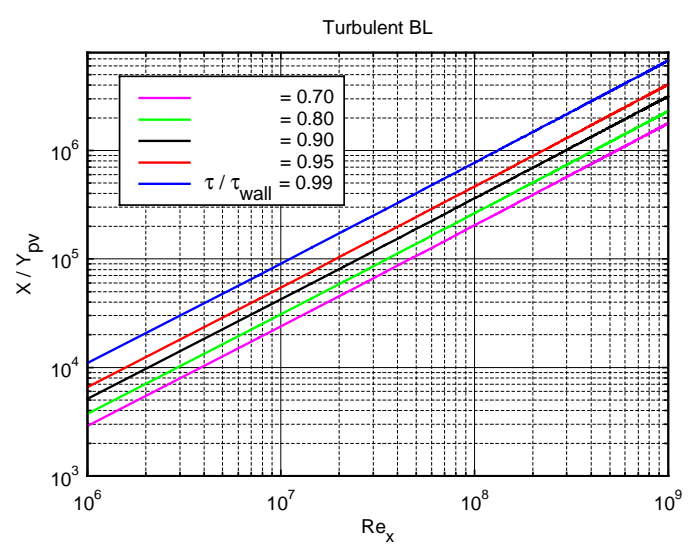

Figure 13. Normalized height above the plate versus Reynolds number.

\section{Measurements at the Large Cavitation Channel, Carderock Division}

The miniature laser Doppler anemometer (MLDA) and two wall shear stress sensors were embedded in a two-dimensional hydrofoil ( $2.1 \mathrm{~m}$ chord, $3.0 \mathrm{~m}$ span). The hydrofoil was installed at the US Navy's William B. Morgan Large Cavitations Channel (LCC) and tests were carried out at speeds from 0.5 to $18 \mathrm{~m} / \mathrm{s}$ and at angles of attacks of $-1,0$, and +1 degrees.

The miniature LDA was located on the suction side of the hydrofoil at a mid-chord location. Figure 14 shows the miniature LDA and cables installed in the hydrofoil. It consisted of a single component frequency shifted miniature LDA attached to a traversing carriage, housed within a sealed container. The container window was flush mounted with the airfoil surface. The traversing mechanism controlled the location of the miniature LD A relative to the hydrofoil surface. Boundary layer data were collected by remotely traversing the miniature LDA probe volume from the surface to the free stream region. Data were collected for four flow speeds each at three angles of attack.. 


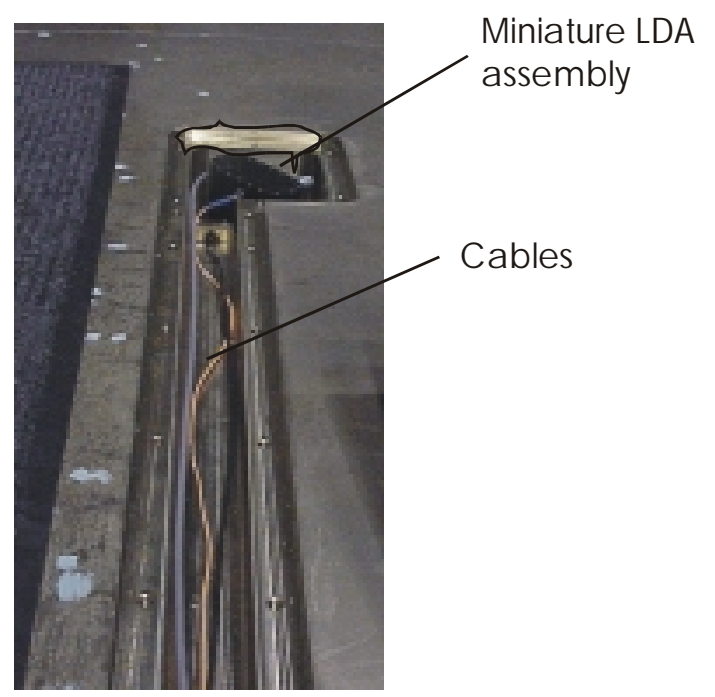

Figure 14. Photograph of the miniature LDA installed in the hydrofoil.

The optical shear stress sensors were flush mounted near the trailing edge of the hydrofoil. One sensor was placed on the suction side and the second sensor was placed on the pressure side of the airfoil. A photograph of one of the sensors mounted flush at the surface of the hydrofoil is shown in Figure 15.

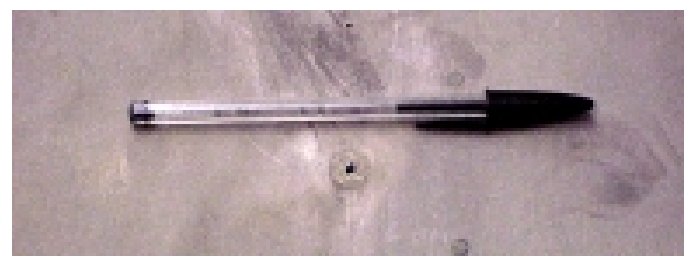

Figure 15. Shear stress sensor mounted flush with the hydrofoil surface.

Figure 16 show the sample velocity profiles obtained at LCC. The precision of the traversing, and the size of the probe volume allowed high resolution definition of the boundary layer very close to the surface. Additional data from these experiments are currently being analyzed.

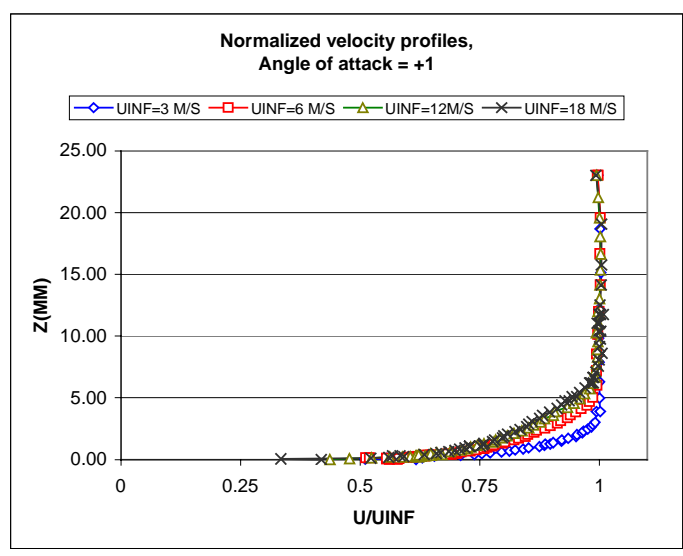

Figure 16. Normalized velocity profiles for +1 degree angle of attack.

\section{Conclusions}

Recent progress in the development of a miniature Laser D oppler Anemometer (LDA) and a micro shear stress sensor were described. Significant steps in the concept development and fabrication of the shear stress sensor stemming from most recent developments in micro device technology were achieved and results obtained in a test facility are reported. The data obtained in a laminar boundary layer with a weak favorable pressure gradient. Perfect agreement between the wall velocity gradient calculated from the boundary layer velocity survey performed with the LD A and the measurements obtained with the shear stress sensor was achieved. The errors associated with the application flow Reynolds number, both for laminar and turbulent flows are presented for the present sensor configuration. The results will serve as an indicator for the applicability of the microshear stress sensor to different flow regimes.

\section{Acknowledgements}

The reported research was supported by ONR, under the Grant number N66001-99-18902 and DARPA and SPAWAR, under the Grant number N66001-99-1-8902 to Caltech. 


\section{$\underline{\text { References }}$}

1. Yeh, Y., and Cummins, H.Z., (1964) "Localized Fluid Flow Measurements with an He-Ne Laser Spectrometer," A ppl. Phys. L ett., Vol. 4, pp. 176-178.

2. Modarress, D. and Johnson, D.A., (1979) "Investigation of Turbulent Boundary Layer Separation Using Laser Velocimetry, A IA A Journal, Vol. 17, No. 7.

3. Lehmann, B., Hassa, C. and Helbig, J., (1996) "Three-Component Laser-D oppler Measurements of the confined Model Flow Behind a Swirl Nozzle," Developments if Laser Techniques and Fluid Mechanics, Selected Papers from the 8th International Symposium, Lisbon, Portugal, Springer Press, 383-398.

4. Modarress D., Tan, (1983) "Application of LDA to Two-Phase Flows," Experiments in Fluids, Vol. 1, pp.129-134.

5. Bachalo, W., (1980) "Method for measuring the size and velocity of spheres by dual-beam light-scatter interferometry," Appl. Optics 3, pp. 363.

6. Dopheide D., Faber M., Yen-Bing-u and Taux G., (1990) "Semiconductor Long-Range Anemometer Using a $5 \mathrm{~mW}$ Diode Laser and a Pin Photodiode", in Applications of Laser Techniques to Fluid Mechanics, R.J Adraian ed., Springer-Verlag, 385-399.

7. Madou, M. (1997) Fundamentals of Microfabrication, CRC Press.

8. Naughton, J.W., Sheplak, M., (2000) "Modern skin friction measurement techniques: D escription, use, and what to do with the data," Paper 2000-2521, 21st AIAA Advanced Measurement Technology and G round Testing, D enver, CO.

9. Naqwi, A. A., Reynolds, W.C., (1987) “D ual cylindrical wave laser-Doppler method for measurement of skin friction in fluid flow,"
Report No. TF-28, Stanford University.

10. Modarress, D., Fourguette, D., Taugwalder, G harib, M, Forouhar, S., Wilson, D., Scalf, J., (2000) "D esign and development of miniature and micro Doppler sensors," 10th International Symposium on Application of Laser Techniques to Fluid Mechanics, Lisbon, Portugal.

11. P. Mouroulis, D. W. Wilson, P. D. Maker, and R. M. Muller, Convex grating types for concentric imaging spectrometers, A ppl. Optics, vol. 37, pp. 7200-7208, Nov. 1, 1998.

12. D. W. Wilson, P. D. Maker, and R. E. Muller, Binary optic reflection grating for an imaging spectrometer," in Diffractive and Holographic Optics Technology III, SPIE Proceedings vol. 2689, Jan. 1996. 